\title{
Pharmacokinetic Analysis of Atractylenolide III in Rat Plasma After Oral Administration of Atractylodes japonica Rhizome Extract by Ultra-Performance Liquid Chromatography-Ion Trap Mass Spectrometry
}

\author{
Jung-Hoon Kim ${ }^{1,2}$ \\ ${ }^{1}$ Division of Pharmacology, School of Korean Medicine, Pusan National University, 50612, Republic of Korea \\ ${ }^{2}$ KM Medicinal Material-based Research Center, School of Korean Medicine, Pusan National University, \\ 50612, Republic of Korea
}

Received: 15 June 2018; accepted: 27 July 2018

\begin{abstract}
Atractylenolide III is one of the major bioactive compounds in Atractylodes japonica rhizome; it has been used clinically for the treatment of gastrointestinal disorders. In the present study, a simple, rapid, and selective analytical method was developed and validated for the quantification of atractylenolide III in rat plasma samples using ultraperformance liquid chromatography-ion trap mass spectrometry (UPLC-ion trap MS). Liquid-liquid extraction with ethyl acetate was used for plasma sample preparation. Bergapten was used as an internal standard (IS). The separation of compounds was carried out on a $\mathrm{C}_{18}$ column, with isocratic elution of $0.1 \%$ formic acid in wateracetonitrile $(45: 55, v / v)$ at $35^{\circ} \mathrm{C}$. Mass detection was performed in the positive ion mode, under optimized conditions for an electrospray ionization source at $\mathrm{m} / \mathrm{z} 249.1$ for atractylenolide III and $\mathrm{m} / \mathrm{z} 217.0$ for the IS. The methods of instrumental analysis and plasma sample extraction were validated in terms of precision, accuracy, matrix effect, and extraction recovery, with acceptable values. The present method was successfully applied to the pharmacokinetic study of atractylenolide III in rat plasma samples after oral administration of $A$. japonica rhizome extract.
\end{abstract}

Keywords: Atractylodes japonica rhizome, atractylenolide III, ultra-performance liquid chromatography-ion trap mass spectrometry, pharmacokinetics, rat plasma

\section{Introduction}

The plant Atractylodes japonica Koidzumi (Asteraceae) is a perennial herb, distributed mostly in Korea, Northeastern China, and Japan. The dried rhizome of A. japonica has been medicinally used for gastrointestinal disorders, as tonifying and replenishing herbal medicinals $[1,2]$. Pharmacological research has demonstrated the improvement of gastrointestinal movement or diseases $[3,4]$.

A. japonica rhizome contains diverse structures of bioactive sesquiterpenes, among which atractylenolide III exhibits antiallergic, antibacterial, anti-inflammatory, and antinociceptive effects [5-8]. As the pharmacological properties of A. japonica rhizome, particularly in in vivo experiments, might be afforded by a diversity of bioactive compounds after oral administration, the pharmacokinetic features of the bioactive compounds need to be evaluated.

Several pharmacokinetic studies of atractylenolides in rat plasma samples after oral administration of an extract of genus Atractylodes rhizomes have been performed using liquid chromatography-triple quadrupole tandem mass spectrometry (LC-MS/MS) or high-performance liquid chromatography (HPLC) [9-12]. In majority of studies, atractylenolides I, II, and III were quantified in rat plasma samples after oral administration of the extract of solely $A$. macrocephala rhizome. However, the pharmacokinetic behavior of atractylenolide III from A. japonica rhizome in animal models, determined using LC-ion trap MS, has not yet been reported.

* Author for correspondence: kmsct@pusan.ac.kr
Therefore, in the present study, a simple, rapid, and selective UPLC-ion trap MS method for the quantification of atractylenolide III in the rat plasma samples after oral administration of the $A$. japonica extract was developed for the first time, and the pharmacokinetic parameters of atractylenolide III were evaluated in in vivo models.

\section{Experimental}

2.1. Materials and Reagents. LC-MS-grade acetonitrile, water, and formic acid were purchased from Fisher Scientific (Pittsburgh, PA, USA). HPLC-grade methanol was obtained from J.T. Baker Inc. (Phillipsburg, NJ, USA). Ethyl acetate was obtained from SK Chemicals (Seongnam, Gyeonggi-do, Korea). Atractylenolide III (purity $\geq 99 \%$ ) was obtained from Biomart (Guangzhou, Guangdong, China). Bergapten (internal standard, purity $\geq 99 \%$ ) was purchased from Chemface (Wuhan, Hubei, China). Dried rhizome of A. japonica was purchased from Kwangmyungdang Medicinal Herbs (Ulsan, Korea) and authenticated by the author. Voucher specimen (2018-PNUKMAJ-01) has been deposited at the School of Korean Medicine, Pusan National University.

2.2. Animals. Male Sprague-Dawley (SD) rats (210-250 g) were supplied by Samtako (Incheon, Korea). Prior to carrying out experiments, the rats were housed at a temperature of $22 \pm 2{ }^{\circ} \mathrm{C}$ and relative humidity of $50 \pm 10 \%$, under a 12 -h light-dark cycle for 7 days, with free access to water. They were then fasted overnight, before administration of the extract. Animal experiments were carried out according to the guidelines for animal experimentation of Pusan National

This is an open-access article distributed under the terms of the Creative Commons Attribution-NonCommercial 4.0 International License (https://creativecommons.org/licenses/by-nc/4.0/), which permits unrestricted use, distribution, and reproduction in any medium for non-commercial purposes, provided the original author and source are credited, a link to the CC License is provided, and changes - if any - are indicated. 
University and the experimental procedures were approved by the Animal Ethics Committee of Pusan National University (PNU-2017-1698).

2.3. Analytical Apparatus. Quantitative analysis was performed using an Accela UPLC system (Thermo Fisher Scientific, San Jose, CA, USA) equipped with a refrigerated autosampler, a degasser, and a quaternary solvent pump coupled with an LCQ Fleet ion trap mass spectrometer (Thermo Fisher Scientific, San Jose, CA, USA). Data were processed using Xcalibur software (v. 2.1.0; Thermo Fisher Scientific, San Jose, CA, USA). Atractylenolide III and internal standard (IS) were separated on a Hypersil GOLD $\mathrm{C}_{18}$ column $(2.1 \mathrm{~mm} \times 50 \mathrm{~mm}, 1.9 \mu \mathrm{m}$; Thermo Fisher Scientific, MA, USA) at $35^{\circ} \mathrm{C}$. The flow rate was $250 \mu \mathrm{L} / \mathrm{min}$, and the injection volume was $5 \mu \mathrm{L}$. The mobile phase consisted of acetonitrile and $0.1 \%$ formic acid in water $(55: 45, v / v)$. Isocratic elution was applied.

An electrospray ionization (ESI) source in the positive ion mode was used for mass spectrometric detection. Mass spectrometric conditions were as follows: sheath gas (nitrogen) $=50 \mathrm{arb}$ units, auxiliary gas (nitrogen) $=30$ arb units, spray voltage $=$ $5.0 \mathrm{kV}$, capillary temperature $=280^{\circ} \mathrm{C}$, and capillary voltage $=$ $35.0 \mathrm{~V}$. Quantitation of the compounds was carried out in the selective ion monitoring (SIM) mode at $249 \mathrm{~m} / \mathrm{z}$ for atractylenolide III and $217 \mathrm{~m} / \mathrm{z}$ for IS.

2.4. Extraction of $A$. japonica Rhizome. Dried rhizomes of $A$. japonica $(500 \mathrm{~g})$ was pulverized and then extracted twice, for $30 \mathrm{~min}$, into $5000 \mathrm{~mL}$ of methanol $(w / v)$ using an ultrasonic extractor (Power Sonic 520; Hwashin Tech, Daegu, Korea). The respective extract was filtered and concentrated using a rotary evaporator (N-3000; Eyela, Miyagi, Japan).

2.5. Preparation of Stock Solutions, Calibration Standards, and Quality Control Samples. To prepare stock solutions, atractylenolide III and IS were accurately weighed and dissolved, separately, in methanol at a concentration of $100 \mu \mathrm{g} / \mathrm{mL}$. The stock solution of atractylenolide III was diluted with methanol to afford the working solutions. IS stock solution was diluted to $2500 \mathrm{ng} / \mathrm{mL}$ with methanol. Calibration standards were prepared by spiking $200 \mu \mathrm{L}$ blank rat plasma with $10 \mu \mathrm{L}$ working solutions and $10 \mu \mathrm{L}$ IS solution. The plasma concentrations of atractylenolide III ranged between 50 and $3200 \mathrm{ng} / \mathrm{mL}$. Quality control (QC) samples were prepared in concentrations of 50,200, and $1600 \mathrm{ng} / \mathrm{mL}$ in the same manner as the plasma calibration standards.

2.6. Plasma Sample Preparation. Plasma samples were prepared using a liquid-liquid extraction (LLE) method. An aliquot of $10 \mu \mathrm{L}$ IS solution and $10 \mu \mathrm{L}$ methanol was added to an aliquot of $200 \mu \mathrm{L}$ plasma sample that had been thawed at room temperature. A volume of $500 \mu \mathrm{L}$ of ethyl acetate was added to this mixture. Extraction of atractylenolide III and IS was achieved after vortex mixing for $2 \mathrm{~min}$ at room temperature. Thereafter, the partitioned mixture was centrifuged at $13,000 \mathrm{rpm}$ for $10 \mathrm{~min}$ at $20^{\circ} \mathrm{C}$. The upper layer was transferred to a clean $1.5-\mathrm{mL}$ polypropylene tube and evaporated using a nitrogen-gas-blowing concentrator (MGS-2200; Eyela, Miyagi, Japan). The residue was reconstituted in $100 \mu \mathrm{L}$ methanol. This was followed by vortex mixing for $1 \mathrm{~min}$ and centrifugation at 13,000 rpm for $5 \mathrm{~min}$ at $20{ }^{\circ} \mathrm{C}$. The supernatant was transferred to an autosample vial, and $5 \mu \mathrm{L}$ of the aliquot was injected into the UPLC-MS/MS system.

\subsection{Method Validation}

2.7.1. Specificity. The specificity was evaluated by comparing chromatograms of blank plasma samples from six different rats, blank plasma samples spiked with atractylenolide III and IS, and plasma samples obtained after intragastric administration of the extracts.
2.7.2. Linearity. The calibration curve was constructed by plotting the peak area ratio of atractylenolide III to IS versus the concentration of atractylenolide III. The linearity was assessed by linear least-squares regression with the weighting factor $1 / x$. The lower limit of quantification (LLOQ) was defined as the lowest concentration in the calibration curve of atractylenolide III, with an acceptance value set at $\pm 20 \%$.

2.7.3. Accuracy and Precision. Six replicates of the QC samples, prepared at three different concentrations of atractylenolide III (100, 400, and $1600 \mathrm{ng} / \mathrm{mL})$, were measured for the assessment of accuracy and precision within a day (intraday precision) and for three consecutive days (inter-day precision). The precision was expressed as the relative standard deviation (RSD\%), and the accuracy was expressed as the relative error (RE\%), with both values accepted within $\pm 15 \%$.

2.7.4. Extraction Recovery and Matrix Effect. The extraction recovery of atractylenolide III was determined by comparing the peak areas obtained from the QC samples with those obtained from the plasma samples spiked with standard solutions post extraction at the same concentration as the QC samples. The matrix effect was determined by comparing the peak areas from the plasma samples spiked with standard solutions after extraction with those from a pure standard solution prepared at the corresponding concentrations.

2.7.5. Stability. The stability of atractylenolide III was measured by analyzing three replicates of three QC samples under different conditions as follows: three freeze-thaw cycles, long-term storage at $-20{ }^{\circ} \mathrm{C}$ for 7 days, short term storage at room temperature for $15 \mathrm{~h}$, and post-preparative storage in an UPLC autosampler for $6 \mathrm{~h}$.

2.8. Pharmacokinetic Study. The extract of $A$. japonica rhizome $(5000 \mathrm{mg} / \mathrm{kg})$ were orally administered to $10 \mathrm{SD}$ rats (male, 210-250 g). Blood samples (approximately $0.4 \mathrm{~mL}$ ) were collected from the jugular vein into heparinized tubes at $0,0.25,0.5,1,1.5,2,3,4,6,8$, and $12 \mathrm{~h}$ after intragastric administration of the extract. The collected blood samples were immediately centrifuged at $13,000 \mathrm{rpm}$ for $10 \mathrm{~min}$. A $200-\mu \mathrm{L}$ volume of supernatant plasma was transferred into a clean tube then stored at $-20{ }^{\circ} \mathrm{C}$ until analysis. The pharmacokinetic parameters of atractylenolide III were calculated with the plasma concentration versus time data by PKSolver, a free add-in program for Microsoft Excel [13].

\section{Results and Discussion}

\subsection{Method Development and Optimization}

3.1.1. Optimization of Analytical Instruments. The optimal ESI conditions were obtained by comparing the ionization of atractylenolide III and IS in both the negative and positive ionization modes through direct infusion to the mass spectrometer. The protonated molecular ions $[\mathrm{M}+\mathrm{H}]^{+}$at $\mathrm{m} / \mathrm{z}$ 249.0 and 217.0 for atractylenolide III and IS, respectively, showed good sensitivity and response (Figure 1).

Optimization of the ESI source was carried out by comparing different conditions such as sheath gas (30-60 arb units), auxiliary gas (10-40 arb units), capillary temperature $\left(250-300{ }^{\circ} \mathrm{C}\right)$, and capillary voltage $(30.0-45.0 \mathrm{~V})$. The highest intensity of protonated molecular ions was obtained under the following conditions: sheath gas 50 arb units, auxiliary gas 30 arb units, capillary temperature $280{ }^{\circ} \mathrm{C}$, and capillary voltage $35.0 \mathrm{~V}$.

Optimal conditions for UPLC were established by comparing the mobile phase modifier and the flow rate to obtain good resolution of atractylenolide III and IS within a short run time. The mobile phase consisting of acetonitrile and 

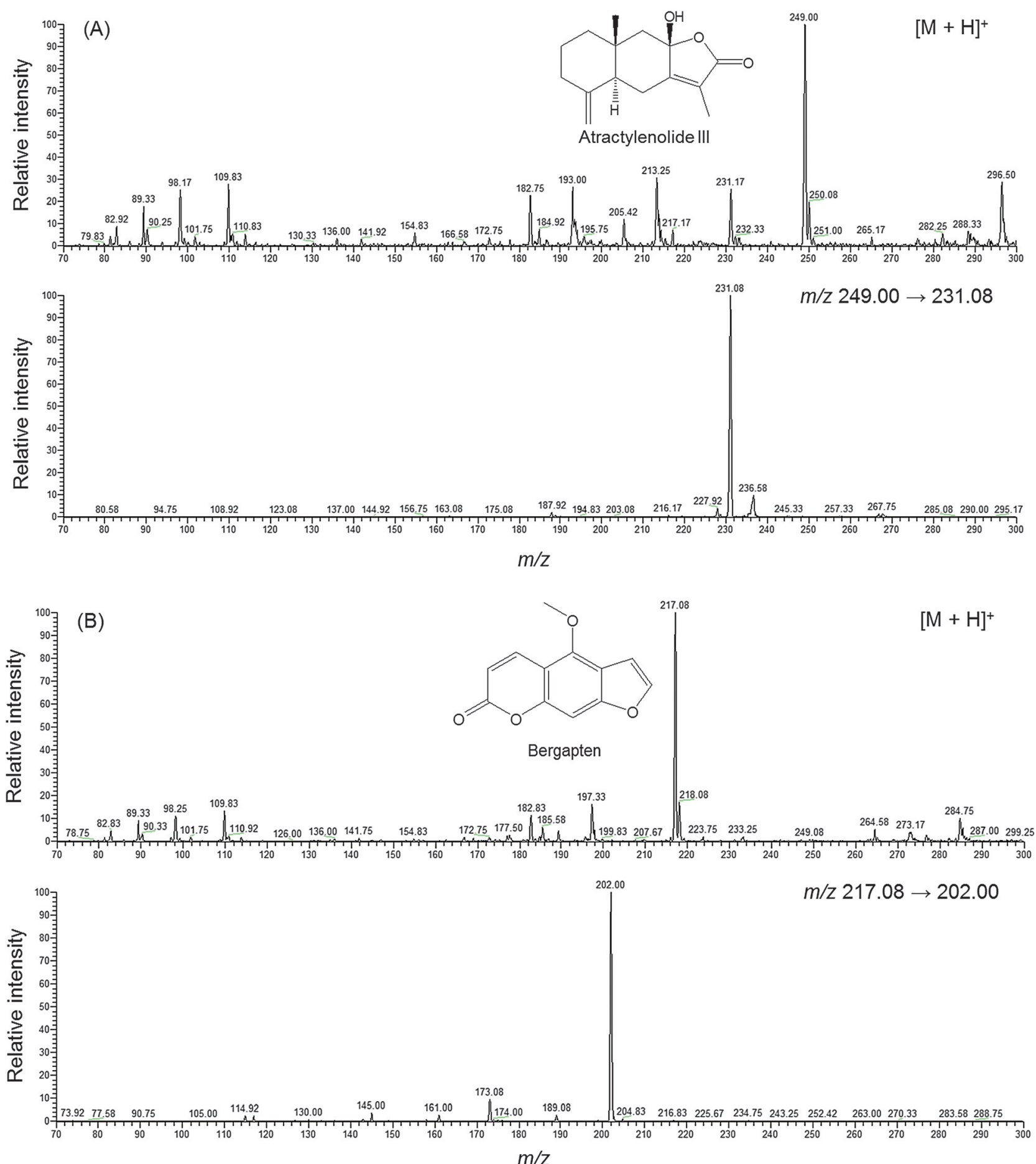

Figure 1. Mass spectra of atractylenolide III (A) and IS (bergapten, B)

$0.1 \%$ formic acid in water, with isocratic elution at the flow rate of $250 \mu \mathrm{L} / \mathrm{min}$, gave better ionization and suitable separation of analytes within $5 \mathrm{~min}$ (1.35 min for atractylenolide III and $0.95 \mathrm{~min}$ for IS).

3.1.2. Optimization of Sample Preparation. Solid-phase extraction (SPE) and LLE were compared for their extraction efficiency of analytes. The cartridge for SPE (Sep-Pak C18; Waters, MA, USA) was used for plasma extraction. The SPE method was found to be very costly and time-consuming, with loss of analyte. LLE was carried out with ethyl acetate in a $1.5-\mathrm{mL}$ polypropylene tube. The LLE method was found to be simple and efficient, and analyte loss was less, as previously reported $[10,12]$. Therefore, LLE with ethyl acetate was chosen as the sample preparation method.

\subsection{Method Validation}

3.2.1. Selectivity. Typical chromatograms were obtained from blank plasma, blank plasma spiked with atractylenolide III and IS, and rat plasma samples obtained from rats after intragastric administration of $A$. japonica extract at $2 \mathrm{~h}$ (Figure 2). The blank plasma showed no interference peak at the retention time of the analytes.

3.2.2. Linearity and $L L O Q$. The calibration curve of atractylenolide III was $y=1.63 \times 10^{-3} x+1.71 \times 10^{-2}$, and the correlation coefficient $\left(R^{2}\right)$ was 0.9975 , which indicates that the curve showed good linearity. The LLOQ for atractylenolide III was $50.00 \mathrm{ng} / \mathrm{mL}$.

3.2.3. Precision and Accuracy. Intra- and inter-day precision (expressed as RSD\%) and accuracy (expressed as RE\%) 

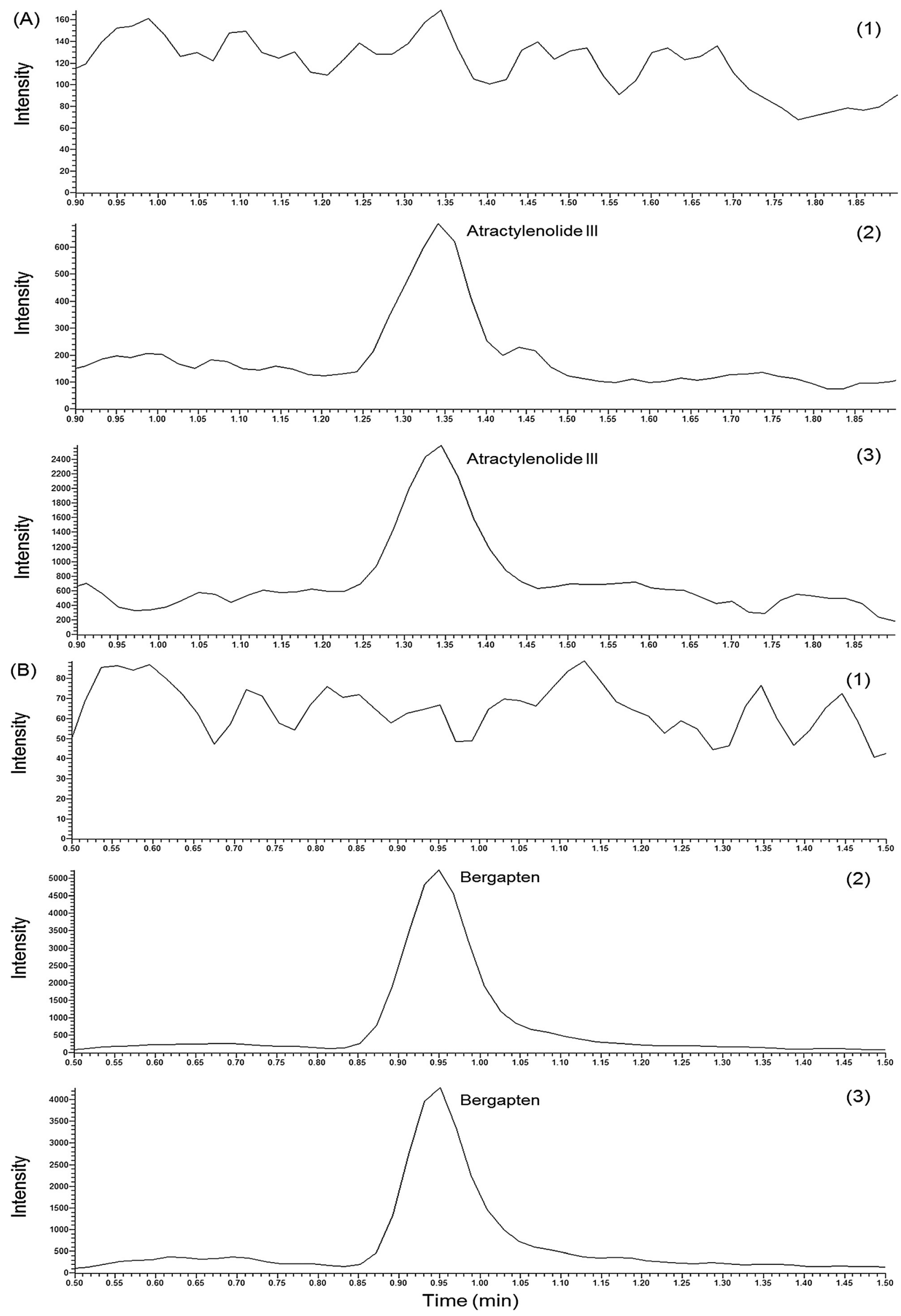

Figure 2. Representative chromatograms of atractylenolide III (A) and IS (B): blank plasma (1), blank plasma spiked with atractylenolide III at LLOQ and IS (2), and plasma samples obtained from rats $2 \mathrm{~h}$ after oral administration of A. japonica extract (3) 
Table 1. Precision and accuracy for atractylenolide III in rat plasma

\begin{tabular}{lccccc}
\hline $\begin{array}{l}\text { Concentration } \\
(\mathrm{ng} / \mathrm{mL})\end{array}$ & \multicolumn{2}{c}{ Intraday $(n=6)$} & & \multicolumn{2}{c}{ Interday $(n=6 \times 3)$} \\
\cline { 2 - 3 } \cline { 5 - 6 } & $\begin{array}{c}\text { Precision } \\
\text { (RSD, \%) }\end{array}$ & $\begin{array}{c}\text { Accuracy } \\
(\mathrm{RE}, \%)\end{array}$ & & $\begin{array}{c}\text { Precision } \\
(\mathrm{RSD}, \%)\end{array}$ & $\begin{array}{c}\text { Accuracy } \\
(\mathrm{RE}, \%)\end{array}$ \\
\hline 100.00 & 10.13 & -0.65 & & 11.53 & -2.87 \\
400.00 & 4.48 & 5.84 & & 6.36 & 3.39 \\
1600.00 & 3.87 & -1.5 & & 3.69 & -0.03
\end{tabular}

$\mathrm{RSD}$, relative standard deviation; RE, relative error.

Table 2. Matrix effect and extraction recovery for atractylenolide III in rat plasma

\begin{tabular}{lccccc}
\hline $\begin{array}{l}\text { Spiked concentration } \\
(\mathrm{ng} / \mathrm{mL})\end{array}$ & \multicolumn{2}{c}{$\begin{array}{c}\text { Matrix effect } \\
(n=6)\end{array}$} & & \multicolumn{2}{c}{$\begin{array}{c}\text { Extraction recovery } \\
(n=6)\end{array}$} \\
\cline { 2 - 3 } \cline { 5 - 6 } & Mean (\%) & RSD (\%) & & Mean (\%) & RSD (\%) \\
\hline 100.00 & 91.13 & 12.68 & & 99.15 & 7.43 \\
400.00 & 109.54 & 4.32 & & 94.17 & 6.00 \\
1600.00 & 110.75 & 2.54 & & 96.88 & 2.09
\end{tabular}

$\mathrm{RSD}$, relative standard deviation; RE, relative error.

of atractylenolide III were within the acceptance range $( \pm 15 \%)$ for the QC samples of different levels. The UPLC-MS method was accurate and reliable for the quantification of atractylenolide III in rat plasma samples (Table 1).

3.2.4. Matrix Effect and Extraction Recovery. The extraction recovery of atractylenolide III was $94.17-99.15 \%$ with RSD $<7.5 \%$, and the matrix effect was $91.13-110.75 \%$ with RSD $<13.0 \%$ over three QC samples (Table 2). These results indicated that the LLE method with ethyl acetate could be used to extract analyte from the plasma sample without much loss and that the matrix effect was not significant for the extraction of the analyte.

3.2.5. Stability. Atractylenolide III in the rat plasma samples appeared stable and none was lost, under the various storage conditions (three freeze-thaw cycles, long period of storage at
$-20{ }^{\circ} \mathrm{C}$, short period of storage at a room temperature, and post-preparative storage in an UPLC autosampler). Hence, an analysis of these plasma samples for a pharmacokinetic study was possible (Table 3).

3.3. Pharmacokinetic Study. The UPLC-MS method established here was validated and successfully adopted in the pharmacokinetic analysis of atractylenolide III in rat plasma samples after intragastric administration of $A$. japonica extract. The mean plasma concentration-time profile of atractylenolide III is shown in Figure 3. The pharmacokinetic parameters, including area under the plasma concentration-time curve (AUC), half-time $\left(t_{1 / 2}\right)$, maximum plasma concentration $\left(C_{\max }\right)$, time to reach the maximum plasma concentration $\left(T_{\max }\right)$, and mean residence time (MRT) are shown in Table 4.

In the mean plasma concentration-time profile of atractylenolide III, a double-peak absorption profile was observed, at $1 \mathrm{~h}$ and $8 \mathrm{~h}$. Several researchers have proposed possible mechanisms for these double absorptions, including (1) enterohepatic recirculation, (2) the presence of absorption sites along the gastrointestinal segments, and (3) variable gastric emptying [14-16].

Besides these mechanisms, it is assumed that interconversion of atractylenolides might occur during metabolism in rats after oral administration. It is reported that atractylenolide II is metabolized to diverse metabolites, mainly hydroxylated or epoxidized products, in rat hepatic microsomes in vitro [17]. Atractylenolide III is produced by oxidation of atractylenolide II in the A. macrocephala rhizome [18]. This oxidation process can also occur in rat liver metabolism, catalyzed by various oxygenases, particularly cytochrome P450 [19], as atractylenolides I, II, and III are all contained in the extract of $A$. japonica rhizome [20]. Further study is required to shed light on the double-peak absorption phenomenon of atractylenolide III after intragastric administration of $A$. japonica extract.

Table 3. Stability of atractylenolide III in rat plasma under different storage conditions $(n=4)$

\begin{tabular}{|c|c|c|c|c|c|c|}
\hline \multirow[t]{3}{*}{ Storage conditions } & \multicolumn{6}{|c|}{ Spiked concentration $(\mathrm{ng} / \mathrm{mL})$} \\
\hline & \multicolumn{2}{|c|}{100.00} & \multicolumn{2}{|c|}{400.00} & \multicolumn{2}{|c|}{1600.00} \\
\hline & $\begin{array}{l}\text { Precision } \\
(\mathrm{RSD}, \%)\end{array}$ & $\begin{array}{c}\text { Accuracy } \\
(\mathrm{RE}, \%)\end{array}$ & $\begin{array}{l}\text { Precision } \\
\text { (RSD, \%) }\end{array}$ & $\begin{array}{c}\text { Accuracy } \\
(\mathrm{RE}, \%)\end{array}$ & $\begin{array}{l}\text { Precision } \\
\text { (RSD, \%) }\end{array}$ & $\begin{array}{c}\text { Accuracy } \\
(\mathrm{RE}, \%)\end{array}$ \\
\hline Three freeze-thaw cycles & 11.17 & 0.30 & 5.15 & 0.02 & 3.40 & 12.74 \\
\hline Storage at $-20^{\circ} \mathrm{C}$ for 7 days & 12.92 & 1.81 & 12.28 & -7.05 & 9.55 & -6.54 \\
\hline Room temperature for $15 \mathrm{~h}$ & 6.98 & 0.03 & 5.1 & -4.21 & 5.35 & 6.84 \\
\hline Processed samples in autosampler for $6 \mathrm{~h}$ & 8.98 & -5.24 & 7.02 & -0.61 & 2.73 & -2.89 \\
\hline
\end{tabular}

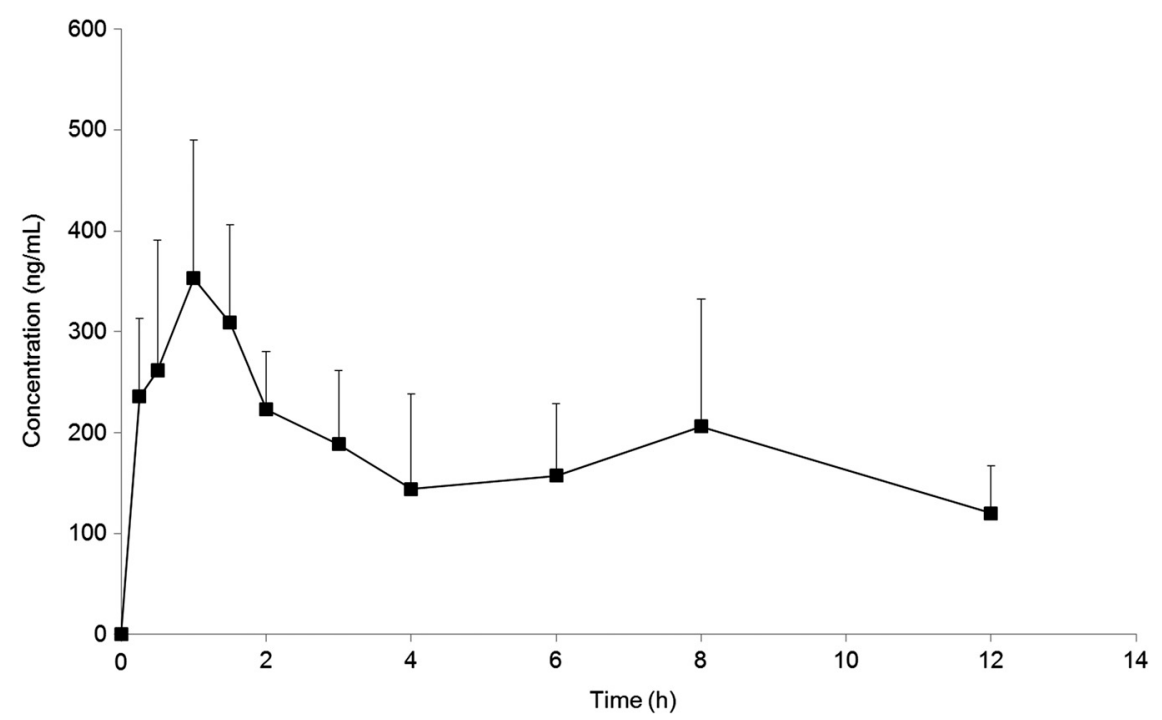

Figure 3. Mean plasma concentration-time curves of atractylenolide III after oral administration of $A$. japonica extract (mean $\pm \mathrm{SD}, n=5$ ) 
Table 4. The pharmacokinetic parameters of atractylenolide III after intragastric administration of $A$. japonica extract $(n=5)$

\begin{tabular}{lc}
\hline Parameters & Mean $\pm \mathrm{SD}$ \\
\hline $\mathrm{AUC}_{0-t}(\mathrm{ng} \cdot \mathrm{h} / \mathrm{mL})$ & $2233.50 \pm 688.07$ \\
$\mathrm{AUC}_{0-\infty}(\mathrm{ng} \cdot \mathrm{h} / \mathrm{mL})$ & $4405.88 \pm 2215.08$ \\
$t_{1 / 2}(\mathrm{~h})$ & $10.74 \pm 6.56$ \\
$C_{\max }(\mathrm{ng} / \mathrm{mL})$ & $392.84 \pm 122.25$ \\
$T_{\max }(\mathrm{h})$ & $1.50 \pm 0.87$ \\
$\mathrm{MRT}_{0-\infty}(\mathrm{h})$ & $16.14 \pm 8.66$ \\
$\mathrm{AUC}_{0-t}$, the area under the curve from 0 time to $12 \mathrm{~h} ; \mathrm{AUC}_{0-\infty}$, the \\
area under the curve from 0 time to infinity; $t_{1 / 2}$, the time taken to drop \\
the drug concentration in plasma to half its value; $C_{\text {max }}$, the maximum \\
drug concentration in plasma; $T_{\text {max }}$, time taken to reach maximum drug \\
concentration in plasma; $\mathrm{MRT}_{0-\infty}(\mathrm{h})$, mean residence time from 0 time \\
to infinity.
\end{tabular}

\section{Conclusion}

A simple, rapid, and selective UPLC-ion trap MS method was developed and successfully applied for the quantification of atractylenolide III in plasma samples from SD rats after oral administration of an extract of $A$. japonica rhizome. The validated analytical method was accurate and precise. The LLE method was efficient, with an ignorable matrix effect. In the present study, the pharmacokinetic parameters of atractylenolide III from $A$. japonica rhizome were determined. The results obtained could serve as a first step toward the further pharmacological application of $A$. japonica rhizome. The present study is the first attempt to establish quantitative analytical methods of atractylenolide III in rat plasma samples after oral administration of $A$. japonica rhizome extract using UPLCion trap MS.

\section{Conflict of Interest}

The author declares that there is no conflict of interest regarding the publication of this article.
Acknowledgement. This work was supported by a National Research Foundation of Korea (NRF) grant (No. 2015R1C1A1A01053466).

\section{References}

1. Kim, J. H.; Kim, Y. S.; Lee, S. H.; Lee, G.; Choi, G.; Jeong, S. I.; Ju, Y. S. Chemical Components of Herbal Medicines in Ungok Herbology Woosuk Press, Jeonju, 2016, pp. 86-87, 218.

2. Korea Food and Drug Administration. Korean Pharmacopeia; The Korea Food and Drug Administration Notification, 2016-147, 2016.

3. Son, H. J.; Jung, K.; Park, Y. H.; Jeon, H. J.; Kang, M.; Ryu, K. H.; Pyo, S. S. Arch. Pharm. Res. 2015, 38, 642-649.

4. Choi, K. H.; Jeong, S. I.; Lee, J. H.; Hwang, B. S.; Kim, S. J.; Lee, S.; Choi, B. K.; Jung, K. Y. Phytomedicine 2011, 18, 408-413.

5. Chen, L. G.; Jan, Y. S.; Tsai, P. W.; Norimoto, H.; Michihara, S.; Murayama, C.; Wang, C. C. J. Agri. Food Chem. 2016, 64, 2254-2262.

6. Hong, M. J.; Kim, E. R.; Kim, J. W. Planta Med. 2012, 78, 74.

7. Zhang, N. N.; Park, D. K.; Park, H. J. J. Ethnopharmacol. 2013, 145, $278-285$.

8. Jeong, S. I.; Kim, S. Y.; Kim, S. J.; Hwang, B. S.; Kwon, T. H.; Yu, K. Y.; Hang, S. H.; Suzuki, K.; Kim, K. J. Molecules 2010, 15, 7395-7402.

9. Li, C. Q.; He, L. C.; Hu, Y. J. Asian J. Pharmacodyn. Pharmacokinet. 2007, 7, 283-288.

10. Shi, Y. Y.; Guan, S. H.; Tang, R. N.; Tao, S. J.; Guo, D. A. Biomed. Chromatogr. 2012, 26, 1386-1392.

11. Li, Y.; Zhang, Y.; Wang, Z.; Zhu, J.; Tian, Y.; Chen, B. J. Pharm. Biomed. Anal. 2012, 58, 172-176.

12. Yan, H.; Sun, Y.; Zhang, Q.; Yang, M.; Wang, X.; Wang, Y.; Yu, Z.; Zhao, Y. J. Chromatogr. B 2015, 993-994, 86-92.

13. Zhang, Y.; Huo, M.; Zhou, J. G.; Xie, S. Comput. Methods Programs Biomed. 2010, 99, 306-314.

14. Wang, Y.; Roy, A.; Sun, L.; Lau, C. E. Drug Metab. Dispos. 1999, 27, $855-859$.

15. Godfrey, K. R.; Arundel, P. A.; Dong, Z.; Bryant, R. Comput. Methods Programs Biomed. 2011, 104, 62-69.

16. Shaw, L. H.; Lin, L. C.; Tsai, T. H. PLoS One 2012, 7, 1-13.

17. Li, Y.; Liu, J.; Wang, X. W. J. Asian Nat. Prod. Res. 2013, 15 344-356.

18. Shan, G. S.; Zhang, L. X.; Zhao, Q. M.; Xiao, H. B.; Zhuo, R. J.; Xu, G.; Jiang, H.; You, X. M.; Jia, T. Z. J. Pharm. Biomed. Anal. 2014, 98, 74-84.

19. Shimada, T.; Mimura, M.; Inoue, K.; Nakamura, S.; Oda, H.; Ohmori, S.; Yamazaki, H. Arch. Toxicol. 1997, 71, 401-408.

20. Cho, H. D.; Kim, U.; Suh, J. H.; Eom, H. Y.; Kim, J.; Lee, S. G.; Choi, Y. S.; Han, S. B. J. Sep. Sci. 2016, 39, 1286-1294. 